\title{
The Potential Usefulness of Virtual Reality Systems for Athletes: A Short SWOT Analysis
}

\author{
Peter Düking ${ }^{1,2 *}$, Hans-Christer Holmberg ${ }^{2,3,4}$ and Billy Sperlich ${ }^{1}$ \\ ${ }^{1}$ Integrative \& Experimental Exercise Science \& Training, Institute for Sport Sciences, University of Würzburg, Würzburg, \\ Germany, ${ }^{2}$ Swedish Winter Sports Research Centre, Mid Sweden University, Östersund, Sweden, ${ }^{3}$ School of Sport \\ Sciences, UiT The Arctic University of Norway, Tromsø, Norway, ${ }^{4}$ School of Kinesiology, University of British Columbia, \\ Vancouver, BC, Canada
}

Keywords: telemedicine, eHealth, mHealth, telerehabilitation, wearable, internet of sports

\section{OPEN ACCESS}

Edited by:

Luca Paolo Ardigò,

University of Verona, Italy

Reviewed by:

David L. Neumann,

Griffith University, Australia

Nicola Luigi Bragazzi,

Università di Genova, Italy

*Correspondence:

Peter Düking

peterdueking@gmx.de

Specialty section:

This article was submitted to

Exercise Physiology,

a section of the journal

Frontiers in Physiology

Received: 19 October 2017

Accepted: 07 February 2018

Published: 05 March 2018

Citation:

Düking $P$, Holmberg $\mathrm{H}-\mathrm{C}$ and

Sperlich B (2018) The Potential Usefulness of Virtual Reality Systems for Athletes: A Short SWOT Analysis.

Front. Physiol. 9:128.

doi: 10.3389/fphys.2018.00128
Virtual reality (VR) systems (Neumann et al., 2017), which are currently receiving considerable attention from athletes, create a two- or three-dimensional environment in the form of emulated pictures and/or video-recordings where in addition to being mentally present, the athlete even often feels like he/she is there physically as well. As she/he interacts with and/or reacts to this environment, movement is captured by sensors, allowing the system to provide feedback.

As with every newly evolving technology related to human movement and behavior, it is important to be aware of the strengths, weaknesses, opportunities and threats (SWOT) associated with the use of this particular type of technology. SWOT analyses are widely utilized for strategic planning of developmental processes (Pickton and Wright, 1998; Tao and Shi, 2016) and it is of great interest to consider whether VR systems should be adopted by athletes or not. Aspects more inherent to the employed technologies of VR systems, and aspects more related to the application of VR systems with athletes are considered as strength/weaknesses and opportunities/threats, respectively. Analogously, SWOT analysis concerning another emerging technology involving sensors of individual parameters (i.e., "implantables") has been performed (Sperlich et al., 2017).

\section{STRENGTHS}

VR systems allow individualization of training (Kim et al., 2013) and can be applied even in everyday settings, such as when traveling, lying in bed or working. Moreover, (bio-)feedback (Düking et al., 2017) can be provided by continuous learning algorithms to athletes directly in real time (Kim et al., 2013) and/or even remotely to coaches (Neumann et al., 2017).

Inherent to the nature of VR is the potential to design and manipulate freely an almost infinite number of procedures for training athletes individually (Hoffmann et al., 2014). For example, manipulation of the visual environment (e.g., fog, light reflections, darkness, dust, rain, snow) allows many different conditions to be experienced. In addition, a large number of repetitions per training session can be achieved, which is likely to be beneficial in connection with sports where this is not possible in real life (e.g., ski jumping, downhill skiing, sky-jumps, and many more). In VR, an individual may compete against or train with any other athlete around the world (Capin et al., 1997; Neumann et al., 2017), regardless of their relative levels of performance, gender, ages and even if the other athlete is injured.

\section{WEAKNESSES}

Realistic environments, which enhance the sense of immersion, are key to optimizing training and learning (Vignais et al., 2015).

The level of immersion depends on the feeling of "being present" in VR (place illusion) and the illusion of what is happening is real (plausibility illusion) (Slater, 2009). 
Consequently, the haptic, tactile, visual, and/or audio (bio-)feedback provided must be as realistic as possible and movements in the real world need to be synchronized with those in the virtual world (Vignais et al., 2015; otherwise, "seasickness" can be induced, Faisal, 2017). However, current VR systems cannot always achieve these goals (Katz et al., 2006).

Moreover, certain VR applications designed to capture the motion of athletes in real time require massive computational power, as well as a broad bandwidth for the transfer of data. Real video footage requires a relatively extensive database, whereas animated video footage may result in the "uncanny valley" effect, i.e., realistic graphical representations of characters that evoke unpleasant feelings (Vignais et al., 2015).

For a more realistic experience, the technology should be non-obtrusive, as small and light-weight as possible, allowing the athlete to execute movements without restriction or harming him/herself or others.

Finally, the costliness of setting up VR systems can limit their usage.

\section{OPPORTUNITIES}

VR systems enable athletes to learn remotely from any coach and at a time and place of their own choosing, improving a wide variety of skills such as decision-making and pacing strategies that optimize utilization of energy (Hoffmann et al., 2014; Murray et al., 2015; Romeas et al., 2015; Gokeler et al., 2016). Creative behavior, involving a wide variety of patterns of movement and tasks (Santos et al., 2016), can be stimulated by providing a plethora of appropriate exercises. Exercising in VR can lower the level of perceived exertion while simultaneously enhancing enjoyment (Mestre et al., 2011), which could increase the willingness to exercise, as well as performance while exercising.

Prior to competitions, VR systems can probably be employed to optimize warm-up procedures (Calatayud et al., 2010), for example, by enhancing motor imagery (Louis et al., 2008). Stress and certain dimensions of (competitive) anxiety could potentially be managed more efficiently with such systems (Parsons and Rizzo, 2008; Stinson and Bowman, 2014). With VR, athletes can train for competitions under the conditions predicted for the actual event, thereby achieving more realistic preparation (Swaren et al., 2012).

VR might also help injured athletes in two ways: First, it could aid the diagnosis of certain aspects of sport-related injuries (Teel and Slobounov, 2015). And secondly, recovery could be promoted by providing exercises designed to maintain mental alertness and readiness through simulation of real-life scenarios from a first-person perspective (Craig, 2014) and/or by helping athletes to maintain appropriate movements during rehabilitation (Fitzgerald et al., 2007; Gokeler et al., 2016).

From an employment perspective, specialized coaches will most likely have to be hired to implement and handle the more complicated VR systems of the future.

For researchers, VR provides exceptional opportunities for highly reliable field-testing of athletes (Gokeler et al., 2016), e.g., their perception-action-loops (Bideau et al., 2010; Craig, 2014).
In the future, such diagnostic tests could also be applied routinely to young athletes, e.g., for earlier identification of talent.

\section{THREATS}

The transferability of skills, tactics, creative behavior and diagnostic procedures from the virtual to the real world remains to be established scientifically, although there is already evidence for the transferability of skills (Tirp et al., 2015). Some VR sensations (e.g., of g-forces, 3-D orientation) are currently not realistic, which could lead to unnatural patterns of movement, as well as under-/overuse and/or injury.

As with every novel technology, VR must first prove its value in order to convince rehabilitation specialists, athletes, coaches and others to adopt it (Katz et al., 2006; Akenhead and Nassis, 2015).

From an economic perspective, certain coaching jobs could be jeopardized by VR systems and, moreover, the cost of certain of these systems is still quite high.

Furthermore, VR systems may pose a threat to certain aspects of health, e.g., mental or visual (Spiegel, 2017). Proper hygiene must be given high priority, especially with respect to avoiding the spread of bacteria and/or viruses among team members (Davies et al., 2017). When exercising in VR, an athlete may be more prone to falling or collision with nearby objects, a risk which appears to be particularly great in connection with visual restriction due to a head-mounted display (Neumann et al., 2017). Another real risk associated with extensive use of VR systems in general is social isolation (Spiegel, 2017).

Finally, the personal data collected by VR systems must be protected from outside access and misuse (Spiegel, 2017).

\section{SUMMARY}

To summarize, VR systems show considerable promise for improving certain aspects of athletic performance, such as tactics or creative behavior, as well as in connection with rehabilitation, and research. Current technological limitations restrict sophisticated application of VR by athletes and transferability from the virtual to the real world and certain related health concerns require detailed further investigation.

Although SWOT analyses have potential limitations (e.g., by being too subjective; Pickton and Wright, 1998), we believe that this opinion article offers a valuable starting point for those who want to know more about the use of VR systems by athletes.

We have pointed out only the most prominent strengths, weaknesses, opportunities and threats associated with the use of VR systems in connection with sports (Table 1) and there are surely many more. It is noteworthy that most current research in this area focuses on aerobic sports and more emphasis on skill-based sports is needed (Neumann et al., 2017). Moreover, VR systems are still in their infancy and the substantial improvements and other alterations certain to come in the near future, as well as the applicability of VR systems to the athletic population must be monitored continuously and carefully. 
TABLE 1 | Strengths, weaknesses, opportunities, and threats associated with the use of VR systems by athletes.

- Individualization of training routines

- Training at any time

- Training in everyday real-life settings

- Biofeedback

- Creation of an almost infinite number of scenarios

- Manipulation of the visual environment

- Repetitive actions not feasible in reality

- Competition against/training with any other athlete regardless of location, age, gender or level of performance

\section{STRENGTHS}

- Limited current presentation of haptic/tactile sensations

- Time lag between the real and virtual worlds

- Requirement for considerable computational power and a broad data bandwidth

- The "uncanny valley" effect

- Limited availability of data

- Obtrusiveness (bulky, heavy equipment)

- Falling or collision with objects

\section{OPPORTUNITIES}

- Remote coaching

- Creation of new job opportunities

- Improvement of skills, tactics and creative behavior

- Preparation for competitions

- Increasing exercise compliance by decreasing perceived exertion and enhancing enjoyment

- Motor imagery, e.g., anticipation of competitive scenarios and warm-up routines

- Management of psychological stress, e.g., fear and anxiety

- Improvement of rehabilitation and recovery by improving diagnostic procedures and allowing training without direct supervision by a therapist

- Reliable research and diagnostic monitoring under realistic conditions, e.g., in the field
- Questionable transferability to real-life scenarios

- Potential development of unnatural patterns of motion

- Non-acceptance by athletes, coaches etc.

- Loss of coaching jobs

- High costs

- Risk to mental and visual health

- Risk of injury due to falling/collision

- Hygienic threats

- Social isolation

- Outside acquisition and misuse of private data

\section{AUTHOR CONTRIBUTIONS}

All authors listed have made a substantial, direct and intellectual contribution to the work, and approved it for publication.

\section{REFERENCES}

Akenhead, R., and Nassis, G. P. (2015). Training load and player monitoring in high-level football: current practice and perceptions. Int. J. Sports Physiol. Perform. 11, 587-593. doi: 10.1123/ijspp.2015-0331

Bideau, B., Kulpa, R., Vignais, N., Brault, S., Multon, F., and Craig, C. (2010). Using virtual reality to analyze sports performance. IEEE Comput. Graph. Appl. 30, 14-21. doi: 10.1109/MCG.2009.134

Calatayud, D., Arora, S., Aggarwal, R., Kruglikova, I., Schulze, S., FunchJensen, P., et al. (2010). Warm-up in a virtual reality environment improves performance in the operating room. Ann. Surg. 251, 1181-1185. doi: 10.1097/SLA.0b013e3181deb630

Capin, T. K., Padnzic, I. S., Noser, H., Thalmann, N. M., and Thalmann, D. (1997). Virtual human representation and communication in VLNet. IEEE Comput. Graph. Appl. 17, 42-53.

\section{FUNDING}

This publication was funded by the German Research Foundation (DFG) and the University of Wuerzburg in the funding programme Open Access Publishing.

Craig, C. (2014). Understanding perception and action in sport: how can virtual reality technology help? Sports Tech. 6, 161-169. doi: $10.1080 / 19346182.2013 .855224$

Davies, H. D., Jackson, M. A., Rice, S. G., Committee on Infectious Diseases, and Council on Sports Medicine and Fitness (2017). Infectious diseases associated with organized sports and outbreak control. Pediatrics 140:e20172477. doi: 10.1542/peds.2017-2477

Düking, P., Holmberg, H. C., and Sperlich, B. (2017). Instant biofeedback provided by wearable sensor technology can help to optimize exercise and prevent injury and overuse. Front. Physiol. 8:167. doi: 10.3389/fphys.2017.00167

Faisal, A. (2017). Computer science: visionary of virtual reality. Nature 551, 298-299. doi: $10.1038 / 551298$ a

Fitzgerald, D., Foody, J., Kelly, D., Ward, T., Markham, C., McDonald, J., et al. (2007). Development of a wearable motion capture suit and virtual reality biofeedback system for the instruction and analysis of sports 
rehabilitation exercises. Conf. Proc. IEEE Eng. Med. Biol. Soc. 2007, 4870-4874. doi: 10.1109/IEMBS.2007.4353431

Gokeler, A., Bisschop, M., Myer, G. D., Benjaminse, A., Dijkstra, P. U., van Keeken, H. G., et al. (2016). Immersive virtual reality improves movement patterns in patients after ACL reconstruction: implications for enhanced criteriabased return-to-sport rehabilitation. Knee Surg. Sports Traumatol. Arthrosc. 24, 2280-2286. doi: 10.1007/s00167-014-3374-x

Hoffmann, C. P., Filippeschi, A., Ruffaldi, E., and Bardy, B. G. (2014). Energy management using virtual reality improves $2000-\mathrm{m}$ rowing performance. $J$. Sports Sci. 32, 501-509. doi: 10.1080/02640414.2013.835435

Katz, L., Parker, J., Tyreman, H., Kopp, G., Levy, R., and Chang, E. (2006). Virtual reality in sport and wellness: promise and reality. Int. J. Comp. Sci. Sport 4, 4-16.

Kim, J., Son, J., Ko, N., and Yoon, B. (2013). Unsupervised virtual realitybased exercise program improves hip muscle strength and balance control in older adults: a pilot study. Arch. Phys. Med. Rehabil. 94, 937-943. doi: 10.1016/j.apmr.2012.12.010

Louis, M., Guillot, A., Maton, S., Doyon, J., and Collet, C. (2008). Effect of imagined movement speed on subsequent motor performance. J. Mot. Behav. 40, 117-132. doi: 10.3200/JMBR.40.2.117-132

Mestre, D. R., Ewald, M., and Maiano, C. (2011). Virtual reality and exercise: behavioral and psychological effects of visual feedback. Stud. Health Technol. Inform. 167, 122-127.

Murray, E. G., Neumann, D. L., Moffitt, R. L., and Thomas, P. R. (2015). The effects of the presence of others during a rowing exercise in a virtual reality environment. Psychol. Sport Exerc. 22, 328-336. doi: 10.1016/j.psychsport.2015.09.007

Neumann, D. L., Moffitt, R. L., Thomas, P. R., Loveday, K., Watling, D. P., Lombard, C. L., et al. (2017). A systematic review of the application of interactive virtual reality to sport. Virtual Real. doi: 10.1007/s10055-017-0320-5. [Epub ahead of print].

Parsons, T. D., and Rizzo, A. A. (2008). Affective outcomes of virtual reality exposure therapy for anxiety and specific phobias: a meta-analysis. J. Behav. Ther. Exp. Psychiatry 39, 250-261. doi: 10.1016/j.jbtep.2007.07.007

Pickton, D. W., and Wright, S. (1998). What's swot in strategic analysis? Strateg. Change 7, 101-109.

Romeas, T., Guldner, A., and Faubert, J. (2015). 3D-Multiple Object Tracking training task improves passing decision-making accuracy in soccer players. Psychol. Sport Exerc. 22, 1-9. doi: 10.1016/j.psychsport.2015.06.002

Santos, S. D., Memmert, D., Sampaio, J., and Leite, N. (2016). The spawns of creative behavior in team sports: a creativity developmental framework. Front. Psychol. 7:1282. doi: 10.3389/fpsyg.2016.01282
Slater, M. (2009). Place illusion and plausibility can lead to realistic behaviour in immersive virtual environments. Philos. Trans. R. Soc. Lond. B. Biol. Sci. 364 3549-3557. doi: 10.1098/rstb.2009.0138

Sperlich, B., Düking, P., and Holmberg, H. C. (2017). A SWOT analysis of the use and potential misuse of implantable monitoring devices by athletes. Front. Physiol. 8:629. doi: 10.3389/fphys.2017.00629

Spiegel, J. S. (2017). The ethics of virtual reality technology: social hazards and public policy recommendations. Sci. Eng. Ethics. doi: 10.1007/s11948-017-9979-y. [Epub ahead of print].

Stinson, C., and Bowman, D. A. (2014). Feasibility of training athletes for highpressure situations using virtual reality. IEEE Trans. Vis. Comput. Graph. 20, 606-615. doi: 10.1109/TVCG.2014.23

Swaren, M., Supej, M., Eriksson, A., and Holmberg, H. C. (2012). "Treadmill simulation of Olympic cross-country ski tracks," in 2nd International Congress on Science and Nordic Skiing (Vuokatti).

Tao, Z. Q., and Shi, A. M. (2016). Application of Boston matrix combined with SWOT analysis on operational development and evaluations of hospital development. Eur. Rev. Med. Pharmacol. Sci. 20, 2131-2139.

Teel, E. F., and Slobounov, S. M. (2015). Validation of a virtual reality balance module for use in clinical concussion assessment and management. Clin. J. Sport Med. 25, 144-148. doi: 10.1097/JSM.0000000000000109

Tirp, J. C. S., Wattie, N., Baker, J., and Schorer, J. (2015). Virtual realities as optimal learning environment in sport - A transfer study of virtual and real dart throwing. Psychol. Test Assess. Model. 57, 57-69.

Vignais, N., Kulpa, R., Brault, S., Presse, D., and Bideau, B. (2015). Which technology to investigate visual perception in sport: video vs. virtual realityHum. Mov. Sci. 39, 12-26. doi: 10.1016/j.humov.2014. 10.006

Conflict of Interest Statement: The authors declare that the research was conducted in the absence of any commercial or financial relationships that could be construed as a potential conflict of interest.

Copyright (c) 2018 Düking, Holmberg and Sperlich. This is an open-access article distributed under the terms of the Creative Commons Attribution License (CC $B Y)$. The use, distribution or reproduction in other forums is permitted, provided the original author(s) and the copyright owner are credited and that the original publication in this journal is cited, in accordance with accepted academic practice. No use, distribution or reproduction is permitted which does not comply with these terms. 